\title{
Validação da escala curta de avaliação funcional do desejo sexual feminino
}

\author{
Validation of the female sexual desire \\ functional short scale
}

\author{
Gustavo Fernando Sutter Latorre', Elissa Bobsin², Lucielle Tatiana Kist' ${ }^{3}$, Erica Feio Carneiro Nunes ${ }^{4}$ \\ ${ }^{1}$ Autor para correspondência. Portal Perineo.net, Florianópolis, Santa Catarina, Brasil. ORCID: 0000-0001-9806-9572. gustavo@perineo.net \\ ${ }^{2}$ Faculdade Inspirar, Criciúma, Santa Catarina, Brasil. ORCID: 0000-0002-3509-8285. licabobsin@yahoo.com.br \\ ${ }^{3}$ Faculdade Inspirar, Florianópolis, Santa Catarina, Brasil. ORCID: 0000-0002-2991-7598. luciellekist@yahoo.com.br \\ ${ }^{4}$ Universidade do Estado do Pará. Belém, Pará, Brasil. ORCID: 0000-0002-1274-4686. erica@perineo.net
}

\begin{abstract}
RESUMO | INTRODUÇÃO: A disfunção do desejo sexual feminino é prevalente e impacta negativamente sobre a função sexual e a qualidade de vida, mas não existem escalas funcionais que levem em conta função e disfunção. OBJETIVO: construir e validar uma escala curta para avaliação funcional do desejo sexual feminino. MÉTODO: Correlação dos resultados de função do desejo sexual avaliado pelo Índice de Função Sexual Feminina (FSFI) e a nova escala funcional, em uma amostra via internet da população feminina brasileira em geral por meio do teste T de Student e o coeficiente de Spearman. Curva ROC fomentou a análise de corroboração entre os dados do domínio disfunção do desejo do FSFI com a nova escala de avaliação funcional. RESULTADOS: Metade das mulheres da amostra apresentou disfunção sexual pelo FSFI, sendo que um terço apresentou disfunção do desejo sexual. Houve boa correlação entre os resultados da nova escala e do domínio desejo sexual do FSFI, bem como boa sensibilidade e especificidade do modelo pela curva ROC. CONCLUSÃO: a nova escala curta de avaliação funcional do desejo sexual feminino com base na Classificação Internacional de Funcionalidade, Incapacidade e Saúde pode ser uma ferramenta útil na avaliação da função do desejo sexual feminino.
\end{abstract}

PALAVRAS-CHAVE: Transtorno do desejo sexual hipoativo. Estudos de validação. Mulheres.

\begin{abstract}
INTRODUCTION: Female sexual desire dysfunction is prevalent and negatively impacts sexual function and quality of life, but there are no functional scales that take into account function and dysfunction. OBJECTIVE: To construct and validate a short scale for functional evaluation of female sexual desire. METHOD: Correlation of sexual desire function results assessed by the Female Sexual Function Index (FSFI) and the new functional scale in an internet sample of the Brazilian female population in general through the Student's $t$ test and the Spearman coefficient. ROC curve fostered corroborating analysis between data from the FSFI desire dysfunction domain with the new functional assessment scale. RESULTS: Half of the women in the sample had sexual dysfunction by FSFI, and one third had sexual desire dysfunction. There was a good correlation between the results of the new scale and the FSFI sexual desire domain, as well as good sensitivity and specificity of the model by the ROC curve. CONCLUSION: The new short functional rating scale for female sexual desire based on the International Classification of Functioning, Disability and Health may be a useful tool in assessing the function of female sexual desire.
\end{abstract}

KEYWORDS: Sexual Dysfunctions. Psychological. Validation studies. Women. 


\section{Introdução}

De modo distinto aos demais mamíferos, a sexualidade do ser humano ultrapassa a função biológica, uma vez que causa prazer sem haver relação com o ciclo reprodutivo. Portanto, deve-se pensar a resposta sexual humana sob três perspectivas: a biológica, a psicológica e a social, sem deixar de desconsiderar as interrelações entre elas' ${ }^{1}$.

A sexualidade humana interfere na saúde física e mental, além de ser influenciada por aspectos emocionais, naturais e sociais ${ }^{2}$. Objetivamente, as disfunções sexuais são desordens psicossomáticas que inviabilizam o prazer e/ou o coito durante uma relação sexual, o que tem como consequências aflições pessoais, além de limitações na qualidade de vida e relações interpessoais ${ }^{3}$.

Já o conceito de saúde sexual compreende a reunião dos elementos corporais, emocionais, racionais e sociais do ser sexual, através de recursos que são engrandecedores, bem como potencializam o amor, a comunicação e a personalidade ${ }^{2}$.

A vida sexual de indivíduos com "saúde sexual" é determinada pela associação de quatro sistemas: endocrinológico, neurológico, vascular ${ }^{4}$ e musculoesquelético ${ }^{5}$. Logo, modificações no funcionamento de qualquer um desses sistemas pode acarretar distúrbios na resposta sexual e, consequentemente, disfunção sexual. De modo mais específico, alterações de qualquer uma das fases do ciclo da resposta sexual feminina - desejo, excitação, orgasmo/satisfação e resolução - podem desencadear disfunções sexuais nos domínios desejo, excitação, lubrificação, orgasmo, satisfação ou dor ${ }^{6}$.

Com relação às disfunções sexuais femininas (DSF), sabe-se que o nível de felicidade e a saúde da relação conjugal são diretamente influenciadas pela satisfação sexual do casal, inclusive para a capacidade de orgasmo da mulher ${ }^{7}$. Ainda, o desconhecimento sobre a própria sexualidade, a ausência de informação sobre a fisiologia humana associada à resposta sexual, dificuldades pessoais e, especialmente, entraves na relação conjugal são fatores que influenciam o desenvolvimento de problemas emocionais nas mulheres, o que causa modificações na sua resposta sexual $^{8}$. De fato, a sexualidade e, portanto, as DSF, são relacionadas em grande parte a fatores socioculturais e comportamentais, que impactam negativamente sobre a saúde em geral destas mulheres ${ }^{9}$.

Todo este panorama permite a inferência de que as DSF são multifatoriais e complexas, desde o início do ciclo da resposta sexual, ou seja, da fase do desejo. Ademais, trata-se de problema relevante pela alta prevalência. A disfunção do desejo sexual aflige $6 \%$ das alemãs ${ }^{10}, 32 \%$ das norte-americanas ${ }^{11}, 69 \%$ das australianas $^{12}, 60 \%$ das brasileiras jovens ${ }^{13}$ e $43 \%$ das brasileiras na faixa dos 25-40 anos ${ }^{14}$.

A disfunção da excitação acomete $57,9 \%$ das japonesas $^{15}, 30 \%$ das iranianas ${ }^{16}, 24 \%$ das norte-americanas $^{17}$ e mais da metade das brasileiras de todas as idades $^{13,14}$. A disfunção do orgasmo pode acometer $32 \%$ das japonesas ${ }^{15}, 37 \%$ das iranianas $^{16}$ e quase $60 \%$ das brasileiras ${ }^{13,14}$. Por fim, a dor sexual acomete $1 \%$ das suecas $^{18}, 25 \%$ das turcas $^{19}, 68 \%$ das malaias ${ }^{20}$ e uma em cada três brasileiras ${ }^{13,14}$.

Atualmente, o questionário mais utilizado mundialmente no estudo e avaliação da função e disfunção sexual feminina é o Female Sexual Function Index (FSFI), criado por uma equipe multidisciplinar de pesquisadores em disfunção sexual feminina ${ }^{21}$. O FSFI é simples de analisar e gerenciar. É um questionário de autorresposta, constituído por uma escala algorítmica que consegue analisar cada comando distintamente ou toda sua constituição ${ }^{22,23}$. Recentemente, foi desenvolvida a versão curta do FSFI, com seis questões ${ }^{24}$ e validada para brasileiras de meia-idade ${ }^{25}$.

Apesar de a disfunção do desejo sexual feminino apresentar, por si só, mais de metade das DSF em geral, o tema permanece ainda pouco esclarecido, especialmente do ponto de vista funcional. Apesar de eficiente, e de ser largamente utilizado em todo o mundo, o FSFI é um questionário grande, e que pressupõe certo tempo para sua aplicação. Considerando que a disfunção do desejo sexual feminino é objeto de avaliação e tratamento por parte de profissionais 
de saúde em geral, uma escala clínica curta de avaliação do desejo feminino, não somente da disfunção, mas da função em sí, poderia ter utilidade clínica e científica relevante desde que objetiva, reduzida, simples de interpretar e preencher e que contemplar graus tanto de função quanto de disfunção. A construção e validação desta escala curta foi, por fim, o objetivo do presente ensaio.

\section{Metodologia}

Trata-se de um estudo observacional, descritivo, quantitativo, qualitativo e transversal comparativo para o desenvolvimento e validação de uma versão curta de avaliação do desejo feminino para aplicabilidade clínica, aprovado pelo Comitê de Ética em Pesquisa da Faculdade Inspirar, sob o CAAE 03628118.1.0000.5221.

Para a validação foi utilizada o FSFI em comparação à escala curta para avaliação funcional do desejo sexual feminino, desenvolvida pelos autores e baseada nos cinco graus de disfunção da Classificação Internacional de Funcionalidade, Incapacidade e Saúde (CIF).

A aplicação dos questionários utilizou o ambiente virtual (online), com a intenção de abranger mulheres de todo o Brasil. A aplicação de uma versão online do FSFI foi recentemente validada ${ }^{13}$.

A população do estudo foi constituída por mulheres acima de 18 anos e sexualmente ativas que aceitaram responder ao questionário de forma voluntária, pelo ambiente virtual, capazes de ler e responder ao texto, brasileiras nativas. Não houve critérios de exclusão.

A amostragem foi por conveniência. Os convites foram veiculados em redes sociais e aplicativos de mensagens, explicando brevemente os objetivos do estudo e convidando possíveis interessadas. Em respeito ao princípio da universalidade e livre acesso, outras mulheres que desejaram participar do estudo, mas que não se encaixaram nos critérios de inclusão ou exclusão, puderam participar normalmente, porém seus dados não fizeram parte da análise estatística. Também foram excluídos da análise estatística os questionários de mulheres que entregaram formulários incompletos.

O FSFI tem suas categorias e subitens fundamentados na categorização de disfunção sexual feminina da American Foundation for Urologic Disease (AFUD). Tratam-se de 19 itens que avaliam seis comandos da função sexual: desejo, dor, excitação, lubrificação, orgasmo e satisfação, com ênfase no distúrbio da excitação. Tal categoria é subdividida em dois comandos distintos de lubrificação (quatro itens) e excitação propriamente dita (quatro itens), possibilitando analisar elementos adjacentes, além dos centrais (excitação subjetiva e desejo ${ }^{26}$. Trata-se de um questionário de autorresposta, constituído por uma escala algorítmica que consegue analisar cada comando distintamente ou toda sua constituição. Nas questões 3 a 14 e 17 a 19, a graduação varia de $0-5$, e nas questões 1 , 2,15 e 16, de 1-5. O resultado geral é delimitado pela soma de cada comando multiplicado por seu fator equivalente e pode variar de 2 a 36 . O ponto de corte para se determinar uma boa função sexual é 26,5 , como foi observado no processo de validação desse instrumento numa população feminina com 18 a 74 anos, com e sem disfunção sexua|23.

A Escala Curta de Avaliação Funcional da Disfunção do Desejo Sexual Feminino é composta por cinco graus de função, conforme os cinco graus de disfunção utilizados na CIF (grau 0: 0 a 5 \% de disfunção; grau 1: 6 a 25\% de disfunção; grau 2: 26 a 50\% de disfunção; grau 3: 51 a 95\% de disfunção e grau 4: 95 a $100 \%$ de disfunção)27. Para cada um dos cinco graus, a mulher deve escolher um padrão de desejo sexual que mais se encaixe na sua média de desejo sexual nos últimos anos, sendo considerados disfuncionais os graus 2, 3 e 4 (Quadro 1). 


\begin{tabular}{|c|c|}
\hline \multicolumn{2}{|r|}{$\begin{array}{l}\text { O desejo sexual compreende a vontade de fazer sexo, não necessariamente quando o corpo começa } \\
\text { responder (excitação: lubrificação, sensação de inchaço vaginal, etc), mas apenas a vontade pura, antes mesm } \\
\text { de a excitação do corpo começar a entrar em ação. Qual dos graus abaixo melhor descreve o seu tipo de desej } \\
\text { sexual nos últimos anos? Escolha apenas um número. }\end{array}$} \\
\hline GRAU & Tipo de sintoma \\
\hline 0 & $\begin{array}{l}\text { Desejo espontâneo: } \\
\text { Tenho vontade de sexo que me surge do nada, uma vez ou outra, mesmo sem que eu seja } \\
\text { estimulada ou pense em algo erótico. }\end{array}$ \\
\hline 1 & $\begin{array}{l}\text { Desejo reativo não tátil: } \\
\text { Sinto vontade de sexo SOMENTE quando imagino, vejo ou escuto algo relacionado a sexo. Não } \\
\text { necessito ser tocada para sentir desejo, mas preciso ver, ouvir ou imaginar algo excitante. }\end{array}$ \\
\hline 2 & $\begin{array}{l}\text { Desejo reativo tátil: } \\
\text { Sinto vontade de sexo SOMENTE quando sou tocada. Não preciso estar sendo penetrada, mas } \\
\text { preciso de toques e carícias, em meus genitais ou outras partes do corpo, para começar a sentir } \\
\text { vontade. }\end{array}$ \\
\hline 3 & $\begin{array}{l}\text { Desejo reativo tardio: } \\
\text { Sinto vontade de sexo SOMENTE quando começa o sexo mesmo, com penetração vaginal. Toques } \\
\text { e carícias, mesmo que genitais, não me fazem sentir vontade. }\end{array}$ \\
\hline 4 & $\begin{array}{l}\text { Desejo ausente: } \\
\text { Eu não sinto vontade de sexo. Nem mesmo durante o ato sexual ou quando sou penetrada. Se fosse } \\
\text { por mim, eu preferiria não fazer sexo. }\end{array}$ \\
\hline
\end{tabular}

Os dados foram coletados de forma online. As participantes do estudo acessaram a plataforma virtual enviada através de e-mail e aplicativo de mensagens para terem acesso ao material da pesquisa. Na primeira página, estava disponível uma breve explicação sobre o estudo e o Termo de Consentimento Livre e Esclarecido, somente após assinatura desde poderia dar-se prosseguimento ao preenchimento.

Cada participante preencheu então as seguintes informações no ambiente virtual: idade, estado civil, orientação sexual, uso de medicamentos antidepressivos, uso de medicamentos anticoncepcionais e/ ou reposição hormonal, esteroides anabolizantes e drogas ilícitas. Em seguida, respondiam a versão da Escala Curta de Avaliação Funcional da Disfunção do Desejo Sexual Feminino e o FSFI.
Ao final, as participantes foram informadas sobre a ocorrência ou não da disfunção do desejo sexual e sobre a função da fisioterapia pélvica no tratamento deste distúrbio, através de um e-mail informativo.

Os dados online foram transferidos para o software estatístico SPSS v.20. Os testes estatísticos utilizados foram o T de Student, o coeficiente de Spearman e curva ROC.

\section{Resultados}

Ao final do estudo, 504 mulheres responderam aos questionários, das quais todas eram sexualmente ativas. A média etária da amostra foi de $28 \pm 12$ anos. A Tabela 1 mostra um panorama geral dos dados sociodemográficos e ginecológicos da amostra. 
Tabela 1. Características sociodemográficas e ginecológicas da amostra

\begin{tabular}{|c|c|c|}
\hline Características & $\mathbf{n}$ & $\%$ \\
\hline \multicolumn{3}{|l|}{ Idade (anos) } \\
\hline $18-27$ & 113 & 22,4 \\
\hline $28-37$ & 213 & 42,3 \\
\hline $38-47$ & 136 & 27 \\
\hline 48 ou mais & 42 & 8,3 \\
\hline \multicolumn{3}{|l|}{ Orientação } \\
\hline Heterossexual & 482 & 95,6 \\
\hline Bissexual & 12 & 2,4 \\
\hline Homossexual & 9 & 1,8 \\
\hline Outros & 1 & 0,2 \\
\hline \multicolumn{3}{|l|}{ Escolaridade } \\
\hline Ens Méd Inc & 2 & 0,4 \\
\hline Ens Méd Comp & 33 & 6,5 \\
\hline Ens Sup Inc & 46 & 9,1 \\
\hline Ens Sup Comp & 423 & 83,9 \\
\hline \multicolumn{3}{|l|}{ Estado Civil } \\
\hline Solteira & 164 & 27,8 \\
\hline Casada/Junta & 340 & 72,2 \\
\hline \multicolumn{3}{|l|}{ Paridade } \\
\hline Nenhum & 189 & 37,5 \\
\hline 1 filho & 175 & 34,7 \\
\hline 2 filhos & 113 & 22,4 \\
\hline 3 ou mais & 26 & 5,2 \\
\hline \multicolumn{3}{|l|}{ Fase Reprodutiva } \\
\hline Gestante & 20 & 4 \\
\hline Puérpera & 67 & 13,3 \\
\hline Menopausa & 34 & 6,7 \\
\hline Outros & 383 & 76 \\
\hline \multicolumn{3}{|l|}{ Atividade Física } \\
\hline Não pratica & 187 & 37,1 \\
\hline $1-3$ vezes/ semana & 247 & 49 \\
\hline +3 vezes/semana & 70 & 13,9 \\
\hline \multicolumn{3}{|l|}{ Substâncias } \\
\hline Antidepressivo & 30 & 6 \\
\hline AC Hormonal & 194 & 38,5 \\
\hline Drogas Ilícitas & 12 & 2,4 \\
\hline Não consome & 268 & 53,2 \\
\hline
\end{tabular}

O uso de antidepressivos apareceu como protetor de DSF ( $p=0,04$ - Pearson), enquanto o uso de anticoncepcionais hormonais e drogas ilícitas estiveram correlacionados à maior frequência de $\operatorname{DSF}(p=0,05)$. Não houve correlação entre a DSF e idade, orientação sexual, escolaridade, estado civil, paridade, fase reprodutiva e prática ou não de atividade física.
As DSF, de acordo com o FSFI, ocorreram em 163 $(32,3 \%)$ mulheres. A disfunção da excitação, caracterizada pela falha na excitação na ausência de disfunção do desejo, esteve presente em 283 (56,1 \%) mulheres. A disfunção da lubrificação esteve presente em $330(65,4 \%)$ mulheres. Já a disfunção do orgasmo, caracterizada pela disfunção do orgasmo 
na ausência de disfunções do desejo e/ou excitação, esteve presente em 19 (3,7\%) das mulheres, enquanto a disfunção da satisfação esteve presente em $456(90,5 \%)$ das mulheres. A dor sexual esteve presente em 115 (22,8\%) mulheres.

A nova escala funcional apresentou boa distribuição dos graus de acordo com o FSFI (Tabela 2). Das 279 mulheres sem DSF pelos critérios do FSFI, 97,5\% foram classificadas dentro dos graus 0 ou 1 (nada ou leve) de disfunção do desejo sexual pela nova escala funcional. Também houve boa distribuição para as mulheres com disfunção do desejo sexual pelo FSFI, sendo que $76 \%$ destas foram classificadas dentro dos graus 2 e 3 (leve ou moderado) da nova escala funcional, e apenas $2,4 \%$ das entrevistadas com DSF do desejo pelo FSFI foram classificadas dentro dos graus 0 e 1 (nada ou leve) da nova escala. A distribuição dos graus da escala nova também foi interessante para o grupo de mulheres com DSF geral (desejo, excitação, lubrificação, orgasmo, satisfação ou dor) pelo FSFI: a maior parte das mulheres (64\%) apresentou graus intermediários (2 ou 3) de disfunção do desejo pela nova escala funcional, enquanto $12,4 \%$ apresentou os graus 0 e 1 (nada ou leve) da escala nova.

Tabela 2. Categorização funcional do tipo de disfunção do desejo funcional ajustada pelo estado de disfunção do desejo sexual e disfunção do desejo sexual pelo FSFI

\begin{tabular}{lcccccc}
\hline & \multicolumn{2}{c}{ Sem DSF* } & \multicolumn{2}{c}{ Com DSF* } & \multicolumn{2}{c}{ Com DDSF* } \\
\hline & $\mathbf{n}$ & $\mathbf{\%}$ & $\mathbf{n}$ & $\mathbf{\%}$ & $\mathbf{n}$ & $\%$ \\
\hline Grau 0 & 158 & 56,6 & 4 & 1,7 & 1 & 0,6 \\
Grau 1 & 114 & 40,8 & 24 & 10,7 & 3 & 1,8 \\
Grau 2 & 5 & 1,8 & 92 & 40,9 & 82 & 50,3 \\
Grau 3 & 2 & 0,7 & 74 & 32,9 & 58 & 35,5 \\
Grau 4 & - & - & 31 & 13,7 & 19 & 11,6 \\
Total & 279 & & 225 & & 163 &
\end{tabular}

*Disfunção Sexual Feminina.

**Disfunção do Desejo Sexual Feminino (DDSF).

O coeficiente de Spearman para o grupo de mulheres com disfunção do desejo sexual pelo FSFI em relação aos escores da Escala Funcional do Desejo foi de $0,78(p=0,01)$, demonstrando correlação significativa entre os dois modelos. O teste qui-quadrado para uma amostra mostrou que também houve diferença significativa entre os graus da nova escala funcional em mulheres com DSF pelo FSFI, quando comparados aos mesmos graus para mulheres sem DSF pelo FSFI $(p=0,000)$. A curva ROC dos escores da Escala Funcional demonstrou boa relação entre a sensibilidade e a especificidade do modelo para explicar a disfunção do desejo sexual feminino pelo FSFI (Figura 1).

Figura 1. Curva ROC entre as variáveis da Escala Funcional para a Disfunção Sexual Feminina ajustada pelo estado de disfunção do desejo sexual pelo FSFI

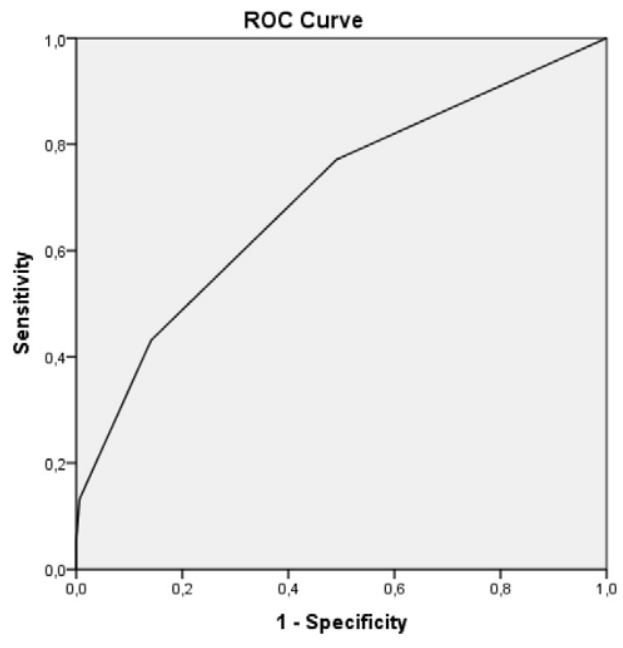

Diagonal segments are produced by ties. 


\section{Discussão}

O presente estudo objetivou validar a Escala Curta de Avaliação Funcional da Disfunção do Desejo Sexual Feminino, comparando seus resultados à do questionário de função sexual feminina FSFI. A primeira impressão a respeito da amostra, composta por muIheres da população em geral e, portanto, sem risco aparente para disfunção sexual, todas sexualmente ativas e com média etária $32 \pm 3$ anos, foi a alta prevalência de disfunção sexual (44,6\% das mulheres). Este valor foi condizente com o apontado por outros estudos sobre a prevalência de DSF na população brasileira em geral 13,14,28.

Considerando a confiabilidade do FSFI como instrumento de avaliação da função sexual feminina, hoje validado e adaptado culturalmente para centenas de línguas e países, essa prevalência permite a conclusão de que a DSF é, hoje, pandêmica, e merece urgente atenção científica, especialmente no tocante ao projeto e implementação de estratégias preventivas e terapêuticas. De fato, urgem medidas para o combate da DSF da população em geral, uma vez que as mulheres com disfunção sexual não buscam ajuda, e que, ainda, os profissionais de saúde em geral não abrem espaço nas consultas para que essas mulheres exponham esse tipo de problema ${ }^{29}$.

Por si só, esses dois fatos já formam a receita do desastre apontado pelos estudos de prevalência supracitados, aos quais se soma, hoje, o presente. Enquanto o interesse científico preocupa-se em explorar quase que exclusivamente mulheres consideradas em risco para a disfunção sexual feminina, como vítimas de câncer, diabetes, sobrepeso, doenças neurológicas, etc., quase metade da população em geral, considerada fora de risco, possui escores compatíveis à disfunção sexual, de acordo com os padrões de avaliação vigentes. Ao final, a pá de cal sobre o argumento acima vem da observação inquietante de que 90,1\% das mulheres (aparentemente saudáveis) que responderam ao presente estudo apresentou escores compatíveis à disfunção da satisfação sexual.

Particularmente, a disfunção do desejo sexual esteve presente em uma em cada três das mulheres da amostra, resultado consonante, novamente, com a literatura nacional ${ }^{13,14}$ e internacional ${ }^{11,30,31}$ para mulheres da população em geral. Particularmente, a disfunção do desejo sexual é preocupante por se tratar de uma disfunção primária ${ }^{6}$, ou seja, que influencia negativamente sobre toda a função sexual subsequente. Noutros termos, uma mulher com disfunção do desejo, muito provavelmente apresentará também degeneração sobre a excitação, orgasmo, satisfação e possivelmente dor, uma vez que o desejo é o gatilho inicial para que toda a resposta sexual ocorra.

O fato de a disfunção do desejo sexual ser primária e, portanto, fundamental na função sexual humana, por si só chama atenção e exige um estudo mais intensivo sobre essa variável. Nesse ínterim, a Escala Curta de Avaliação Funcional da Disfunção do Desejo Sexual Feminino vem somar esforços na medida em que fomenta de maneira quali-quantitativa o nível desta disfunção, em graus, de modo prático e voltada à prática clínica diária. Inspirada sobre os valores de degeneração funcional da CIF, a Escala Curta de Avaliação Funcional da Disfunção do Desejo Sexual Feminino apresenta cinco graus crescentes de disfunção, que podem ser reconhecidos pela mulher que, de acordo com os procedimentos da CIF, ela própria deve classificar a si mesma em um dos grupos, sendo moderada pelo profissional de saúde que acompanha sua avaliação ${ }^{27}$.

Restava saber se haveria correlação entre os valores observados pela aplicação da Escala Curta de Avaliação Funcional da Disfunção do Desejo Sexual Feminino e os valores observados pelo domínio de disfunção do desejo sexual feminino do universalmente utilizado questionário FSFI. Tal correlação foi explorada e apresentada, e seus resultados permitiram a inferência de que a escala curta é válida, quando dissecada à luz do FSFI. Pode-se observar que o grupo de mulheres com disfunção do desejo, de acordo com o FSFI, distribuiu-se de maneira quase linear quanto aos graus crescentes da Escala Curta. Poucas mulheres $(2,4 \%)$ com disfunção do desejo de acordo com FSFI apresentaram os graus mais brandos (0 e 1) de disfunção do desejo de acordo com a Escala Curta. Quanto à sensibilidade, a Escala Curta diferenciou em graus a disfunção do desejo sexual das mulheres com escores compatível à essa disfunção pelo FSFI: houve mulheres distribuídas através de todos os graus (0-4) da Escala Curta, sendo mais prevalentes os graus medianos de disfunção do desejo (graus 2 e 3). A curva ROC mostrou boa relação entre sensibilidade e especificidade da Escala Curta. A soma dessas observações permite a conclusão de que a Escala Curta parece sensível e específica para qualificar e quantificar em graus funcionais a disfunção do desejo sexual feminino, podendo servir de 
ferramenta para avaliação, evolução e acompanhamento da pacientes pós-alta.

Por fim, outras DSF específicas estiveram presentes na amostra estudada. A disfunção da excitação, caracterizada pela falha na excitação na ausência de disfunção do desejo, esteve presente em 283 (56,1 \%) mulheres. A disfunção da lubrificação esteve presente em $330(65,4 \%)$ mulheres. Já a disfunção do orgasmo, caracterizada pela disfunção do orgasmo na ausência de disfunções do desejo e/ou excitação, esteve presente em 19 (3,7\%) das mulheres. Aqui, vale salientar que a queixa de problemas no orgasmo é diferente da disfunção do orgasmo. Pela definição, disfunção do orgasmo ${ }^{6}$ é caracterizada por queixas de problemas no orgasmo (prematuro, retardado ou fraco) na ausência de disfunções do desejo e da excitação. Noutros termos, uma mulher com queixa de orgasmo, mas com desejo ruim deveria ser classificada dentro do conceito de disfunção do desejo, e tratada por este motivo. $O$ mesmo raciocínio vale para mulheres com queixas do período de excitação e durante o intercurso (platô), como problemas na lubrificação, sensibilidade, etc.: mesmo quando apresentam queixas (esperadas) de problemas no orgasmo, estas deveriam ser classificadas e tratadas como portadoras de disfunção da excitação. Portanto, numa amostra estudada por meio do FSFI, o grupo de mulheres com disfunção do orgasmo propriamente dita compreende uma intersecção entre o grupo formado por todas as mulheres que possuem escores de corte abaixo de 5,05 no domínio orgasmo do $\mathrm{FSFI}^{23}$, menos os grupos de mulheres com escores inferiores a 4,28 no domínio desejo, o de mulheres com menos de 5,28 pontos no domínio excitação e aquelas com menos de 5,08 pontos no domínio lubrificação, para que a definição de disfunção do orgasmo seja respeitada. Realizadas essas operações, poucas mulheres, de fato, apresentaram disfunção do orgasmo genuína, apesar de grande parte da amostra apresentar, de modo previsível, queixas de orgasmo. Afinal, como seria a função orgástica de uma mulher com falha no desejo e/ou excitação e/ou lubrificação, senão também falha?

\section{Conclusão}

A disfunção do desejo sexual feminino é prevalente e impacta de modo relevante sobre a vida sexual e assim a qualidade de vida de mulheres por todo o mundo. Ante a necessidade de avaliar de modo funcional e escalar a severidade da disfunção do desejo sexual, uma escala curta de avaliação funcional do desejo sexual feminino foi desenvolvida a partir da Classificação Internacional de Funcionalidade, Incapacidade e Saúde, e comparado aos resultados do questionário FSFI.

De modo geral foi alta a prevalência de disfunção sexual nas mulheres estudadas, praticamente metade da amostra. De modo específico, a disfunção do desejo sexual feminino também se mostrou relevante, aparecendo em um terço das mulheres, dados em consonância com a prevalência descrita na literatura nacional e internacional. Houve boa correlação entre os valores da escala curta e o domínio do FSFI que mede a disfunção do desejo sexual, demonstrando boa sensibilidade e especificidade do novo instrumento.

A escala curta de avaliação funcional do desejo sexual feminino pode servir como instrumento complementar para a avaliação quali-quantitativa da função e da disfunção do desejo sexual, com aplicabilidade clínica e de forma prática, tanto na avaliação, quanto na evolução, alta e acompanhamento pós-alta de pacientes tratados por este distúrbio.

\section{Contribuições dos autores}

Latorre GFS participou da concepção, delineamento, busca e análise estatística dos dados da pesquisa, interpretação dos resultados, redação do artigo científico. Bobsin ES participou da coleta de dados da pesquisa e redação do artigo científico. Kist LTL participou da coleta de dados da pesquisa e redação do artigo científico. Nunes EFC participou da interpretação dos dados e revisão do artigo científico.

\section{Conflitos de interesses}

Nenhum conflito financeiro, legal ou político envolvendo terceiros (governo, empresas e fundações privadas, etc.) foi declarado para nenhum aspecto do trabalho submetido (incluindo, mas não se limitando a subvenções e financiamentos, participação em conselho consultivo, desenho de estudo, preparação de manuscrito, análise estatística, etc.). 


\section{Referências}

1. Vittielo N, Rodrigues Junior OMR. As bases anatômicas e funcionais do exercício da sexualidade. São Paulo: Iglu; 1997.

2. Organização Mundial de Saúde. Instruccion y asistencia en cuestiones de sexualidade humana: formacion de profisionales de la Salud. Genebra; 1975.

3. Kaplan HS. A nova terapia do sexo. 6.ed. São Paulo: Nova Fronteira; 1974.

4. Penson RT, Gallagher J, Gioiella ME, Wallace M, Borden K, Duska LA. Sexuality and cancer: conversation comfort zone. Oncologist. 2000;5(4):336-44. doi: 10.1634/theoncologist.5-4-336

5. Puppo V. Anatomy and Physiology of the Clitoris, Vestibular Bulbs, and Labia Minora With a Review of the Female Orgasm and the Prevention of Female Sexual Dysfunction. Clin Anat. 2013;26(1):134-152. doi: 10.1002/ca.22177

6. Basson R, Althof S, Davis S, Fugl-Meyer K, Goldstein I, Leiblum S et al. Summary of the Recommendations on Sexual Dysfunctions in Women. J Sex Med. 2004;1(1):24-34. doi: 10.1111/j.1743$\underline{6109.2004 .10105 . x}$

7. Diamantino EMV, Clímaco FMS, Ajzman JC, Nowak LD, Oliveira LDP, Barreto RHA et al. Aspectos básicos da sexualidade humana na prática clínica: parte II. Femina. 1993;21(11):1152-80.

8. Phillips NA. Female sexual dysfunction: evaluation and treatment. Am Fam Physician. 2000;62(1):127-36, 141-2.

9. Abdo C. Sexualidade humana e seus transtornos. 3.ed. São Paulo: Leitura Médica; 2010.

10. Wallwiener CW, Wallwiener LM, Seeger H, Mück AO, Bitzer J, Wallwiener M. Prevalence of sexual dysfunction and impact of contraception in female German medical students. J Sex Med. 2010;7(6):2139-2148. doi: 10.1111/j.1743-6109.2010.01742.x

11. Shindel AW, Ferguson GG, Nelson CJ, Brandes SB. The sexual lives of medical students: a single institution survey. J Sex Med. 2008;5(4):796-803. doi: 10.1111/j.1743-6109.2007.00744.x

12. Worsley R, Bell RJ, Gartoulla P, Davis SR. Prevalence and Predictors of Low Sexual Desire, Sexually Related Personal Distress, and Hypoactive Sexual Desire Dysfunction in a Community-Based Sample of Midlife Women. J Sex Med. 2017;14(5) 675-686. doi: 10.1016/j.jsxm.2017.03.254

13. Latorre GFS, Bilck PA, Cardoso FL, Sperandio FF. Validade e confiabilidade de uma versão on-line do Female Sexual Function Index por teste e reteste. Rev Bras Ginecol Obstet. 2013;35(10):469-474. doi: 10.1590/S0100-72032013001000008
14. Antônio JZ, Silva A, Costa PPB, Jung D, Pereira CF, Nunes EFC et al. Função sexual feminina, desgaste emocional por insatisfação sexual e inteligência emocional. Fisioter Bras 2016;17(6):544-550.

15. Hisasue S, Kumamoto Y, Sato $Y$, Masumori N, Horita H, Kato $\mathrm{R}$ et al. Prevalence of female sexual dysfunction symptoms and its relationship to quality of life: a Japanese female cohort study. Urology. 2005;65(1):143-8. doi: 10.1016/j.urology.2004.08.003

16. Safarinejad MR. Female sexual dysfunction in a populationbased study in Iran: prevalence and associated risk factors. Int J Impot Res. 2006;18(4):382-95. doi: 10.1038/sj.ijir.3901440

17. Jamieson DJ, Steege JF. The prevalence of dysmenorrhea, dyspareunia, pelvic pain, and irritable bowel syndrome in primary care practices. Obstet Gynecol. 1996;87(1):55-58. doi: 10.1016/0029-7844(95)00360-6

18. Shindel AW, Ferguson GG, Nelson CJ, Brandes SB. The sexual lives of medical students: a single institution survey. J Sex Med. 2008;5(4):796-803. doi: 10.1111/j.1743-6109.2007.00744.x

19. Cayan S, Akbay E, Bozlu M, Canpolat B, Acar D, Ulusoy E. The prevalence of female sexual dysfunction and potential risk factors that may impair sexual function in Turkish women. Urol Int. 2004;72(1):52-7. doi: 10.1159/000075273

20. Sidi H, Puteh SE, Abdullah N, Midin M. The prevalence of sexual dysfunction and potential risk factors that may impair sexual function in Malaysian women. J Sex Med. 2007;4(2):311-21. doi: $10.1111 /$ j.1743-6109.2006.00319.x

21. Rosen RC, Brown C, Heiman J, Leiblum S, Meston C, Shabsigh $\mathrm{R}$ et al. The female sexual function index (FSFI): a multidimensional self-report instrument for the assessment of female sexual function. J Sex Marital Ther. 2000;26(2):191-208. doi: $\underline{10.1080 / 009262300278597}$

22. Meston CM. Validation of the Female Sexual Function Index (FSFI) in women with female orgasmic disorder and in women with hypoactive sexual desire disorder. J Sex Marital Ther. 2003;29(1):39-46. doi: 10.1080/713847100

23. Wiegel M, Meston C, Rosen R. The female sexual function index (FSFI): cross-validation and development of cutoff scores. J Sex Marital Ther. 2005;31(1):1-20. doi: $10.1080 / 00926230590475206$

24. Dall'Agno ML, Ferreira CF, Ferreira FV, Pérez-López FR, Wender MCO. Validation of the Six-item Female Sexual Function Index in Middle-Aged Brazilian Women. Rev Bras Ginecol Obstet. 2019;41(7):432-439. doi: 10.1055/s-0039-1692694

25. Isidori AM, Pozza C, Esposito K, Giugliano D, Morano S, Vignozzi $L$ et al. Development and validation of a 6-item version of the female sexual function index (FSFI) as a diagnostic tool for female sexual dysfunction. J Sex Med. 2010;7(3):1139-1146. doi: 10.1111/j.1743-6109.2009.01635.x 
26. Pacagnella RC, Vieira EM, Rodrigues Junior OM, Souza C. Adaptação transcultural do Female Sexual Function Index. Cad Saúde Pública. 2008;24(2):416-426. doi: 10.1590/S0102$\underline{311 \times 2008000200021}$

27. Organização Mundial de Saúde. Classificação Internacional de Funcionalidade, Incapacidade e Saúde - CIF. Lisboa: OMS; 2004.

28. Abdo CH, Oliveira WM Jr, Moreira ED Jr, Fittipaldi JAS.

Prevalence of sexual dysfunction and correlated conditions in a sample of brazilian women: results of the Brazilian study on sexual behavior (BSSB). Int J Impot Res. 2004;16(2):160-6. doi: 10.1038/sj.ijir.3901198

29. Cumming GP, Currie HD, Moncur R. The effects of urinary incontinence and availability of publically accessible toilets: an online survey. Menopause Int. 2011;17(1):14-5. doi: 10.1258/ mi.2011.011004

30. Komboigo BE, Kiemtoré $S$, Kain DP, Zamané $Y$, Kaboré $X$, Zoundi $\mathrm{M}$ et al. Evaluation of sexual dysfunctions among women living with a partner in Ouagadougou, Burkina. Med Sante Trop. 2019;29(3):310-316. doi: 10.1684/mst.2019.0932

31. Maasoumi R, Elsous A, Hussein $H$, Taghizadeh Z, Baloushah $S$. Female sexual dysfunction among married women in the Gaza Strip: an internet-based survey. Ann Saudi Med. 2019;39(5):319327. doi: $10.5144 / 0256-4947.2019 .319$ 